\title{
STABILITY OF ATOMS IN INTENSE LASER FIELDS
}

\author{
P. H. Bucksbaum and R.R. Jones \\ Department of Physics, University of Michigan, Ann Arbor, MI 48109-1120
}

\begin{abstract}
Several mechanisms can impede or even eliminate photoionization or photodissociation of bound quantum systems in an intense laser field. This paper reviews recent laboratory studies of these effects.

\section{INTRODUCTION}

When laser fields become comparable to the static fields in atoms, multiphoton absorption is very rapid. Multiphoton ionization and Above-threshold ionization dominate the landscape of experimental atomic physics in this regime. ${ }^{1}$ Despite the seeming inevitability of photoionization in such extreme environments, recent theories suggest that atoms and molecules may remain stable under some circumstances. ${ }^{2,3}$ The physical origins of this effect can be found in classical ideas about dynamical stabilization in the presence of a periodic time-varying force, as well as in quantum-mechanical concepts of interference. In this paper we will discuss the stabilization of bound quantum systems at high intensities from an experimental perspective.
\end{abstract}

\section{STABILIZATION}

2.1 Dichotomy The case for stable states has been tied to the notion of wavefunction distortion in the atom. ${ }^{2}$ To understand this, first visualize the motion of a classical electron in the presence of a monochromatic radiation field. If we boost into the frame where the electron is at rest on time average, we find that the particle executes a "figure-8" motion due to the combined effect of the electric and magnetic field. This is spoiled only by re-radiation (i.e. linear and nonlinear Compton scattering) which leads to damping or to light-pressure acceleration. Those effects are so small as to be quite unimportant.

The figure- 8 lies in the plane containing the polarization $\hat{\varepsilon}$ and the laser momentum vector $\mathbf{k}$. The intensity where atoms usually become unstable for optical frequency laser light $(\mathrm{h} v \sim 2 \mathrm{eV})$ is $\mathrm{I}<10^{14} \mathrm{~W} / \mathrm{cm}^{2}$, or peak laser electric field $\mathrm{F}_{\mathrm{o}}-3 \mathrm{~V} / \AA$. Although sufficient to tear most atoms apart, this intensity is still low enough to neglect the force exerted by the magnetic field, so the free electron merely oscillates in one dimension along $\hat{\varepsilon}$ with an amplitude of about $6 \AA$, and a frequency equal to the laser frequency.

Now, imagine that we turn the laser frequency and intensity up together, in such a way that the amplitude of this wiggle motion remains roughly constant -- in other words, we just speed up the movie. Eventually the force exerted by the light becomes so high that it overwhelms a force that would be exerted by the Coulomb potential of 
a nucleus inserted into the electron's orbit. The electron in such an atom is nearly free, except for a small "anchor" force exerted by the nucleus. In this simple classical picture, ionization is the result of Coulomb scattering from the nucleus as the electron speeds past. Since this scattering cross section decreases as $1 / \mathrm{E}^{2}$, the atom becomes more stable as the intensity increases. This completes a simple classical picture of stabilization.

A rigorous quantum theory of this type of stabilization shows several interesting additional features. These calculations ${ }^{2}$ use a number of special techniques such as transformation of coordinates into a non-inertial wiggling "Kramers" frame. Unfortunately we cannot take space to describe the details here. One of the important results, however, is that the wave function of the ground state of hydrogen in this high frequency, high intensity limit undergoes a bifurcation into two probability amplitude maxima. These are separated by the classical wiggle amplitude. This splitting has been termed "dichotomy" by its discoverers. ${ }^{2}$ A second important result is that the total binding energy of the atom goes down, roughly as the square root of the intensity. (Recall that in conventional ATI, the binding energy of the ground state of an atom increases linearly with intensity, by an amount equal to the Ponderomotive Potential $U_{p} . U_{p}$ is the classical wiggle energy of a free electron in the field, and is approximately equal to $\lambda^{2} \mathrm{IeV}$, where the wavelength, $\lambda$, is expressed in $\mu \mathrm{m}$ and the intensity, $\mathrm{I}$, is in units of $10^{13} \mathrm{~W} / \mathrm{cm}^{2}$.

The most important prediction for our purposes is that the lifetime of the state, which was rapidly decreasing with intensity in the perturbative and ATI regimes, actually increases with intensity in the dichotomous region. Thus, the atom becomes stable.

\subsection{Stabilization of Rydberg States A second type of stabilization occurs for Rydberg} states, and was introduced by Federov and coworkers. ${ }^{3}$ They considered the strong coupling between a Rydberg state and the continuum for high frequency light. (High frequency in this case just means that one-photon transitions between the Rydberg state and the continuum are possible.) The lifetime of the state in the low intensity limit decreases as $1 / \mathrm{I}$, of course. The eigenenergies therefore take on a width proportional to I. At some point, neighboring states will begin to overlap. This happens when the ionization rate approaches the classical orbit rate for that state. In this intensity range the atom is maximally unstable -- the Rydberg states in question really merge with the continuum. However, at still higher intensities, stability is restored! This has been described in various ways. One view is that interferences develop between neighboring Rydberg states once the states overlap in energy. The overlapped states can couple to each other via stimulated Raman scattering through the continuum, and this scattering leads to the formation of new stable states in the Rydberg/continuum manifold. These new states have energies intermediate between the original Rydberg eigenvalues, because they really represent superpositions of the original states. 
The formation of non-ionizing superposition states has an analog in the time domain: A very short laser pulse can excite many Rydberg states simultaneously. These are localized wavepackets of probability amplitude, which execute classical Kepler orbits around the ion core. Such superposition states have vanishing ionization cross sections during the parts of their orbit when they are away from their parent ion. ${ }^{5,6}$ Wavepacket behavior is periodic -- as the wavepacket returns to the ion, it may ionize. Federov's intensity-stabilized states do not exhibit this periodicity, but rather remain stable so long as the intense laser is present.

The reader who is dissatisfied with these brief descriptions of high intensity stabilization is encouraged to consult the original literature on the subject. We will now return to the experimental issues which form our main subject.

\section{GENERIC PROBLEMS FOR EXPERIMENTS SEEKING STABILIZATION}

3.I High Frequency Limit Experiments seeking evidence for stabilization must first employ sufficiently high frequency and high intensity light, as implied by the theories in the previous section. For example, if the ground state of hydrogen is to become "dichotomous," one requires first a laser with a frequency above one Rydberg!. Most high intensity lasers operate in the visible and near infrared; a few, such as $\mathrm{KrF}$ and ArF excimers, operate in the near ultraviolet.

Fortunately, there are several ways around this wavelength mismatch. First, excited states of atoms can always be found where the binding is much less that the available laser photon energy. The Rydberg stabilization should therefore be observable with present-day lasers.

Even dichotomy might be achieved if the magnetic quantum number of the initial state is sufficiently high. Since the laser can be polarized to only couple states of the same $m$, the lowest such state, with principal quantum number $m+1$, is in effect a ground state for that system.

Finally, one may take advantage of the reduced ionization potential in the stabilization regime. Once the binding energy drops far below the energy of an optical photon, lasers at that photon energy are in the high frequency limit. The trick is getting to that regime without ionizing first.

3.2 Death Valley In an experiment, atoms are subjected to a range of laser intensities. As the laser intensity increases the atom will experience lower intensities first. All theories predict a range of intermediate intensities where the lifetime is at a minimum, before stabilization sets in. This dip in the lifetime vs. intensity has been termed "Death Valley," and experiments must meet the challenge of traversing this dangerous landscape in order to get to more stable ground on the other side.

The depth of the Death Valley minimum depends on several factors. For dichotomous stabilization of atomic states with $m \geq 6$, the dip is shallow enough that subpicosecond turn-on times might allow the atom to survive. In the high frequency 
limit, the dip is also shallower. However, for Rydberg states the minimum lifetime is really set by a dynamical timescale of the atom itself, the Kepler orbit time. The intensity must ramp up rapidly compared to this time in order to assure at least partial survival of the bound system.

3.3 Flop Out The signature of stabilization is that the atom survives. In other words, we are looking for something, i.e. ionization, not to happen. This is called a "flopout" experiment, an old atomic beams term that describes any experiment where the decrease in signal (ionization signal in this case) indicates the presence of the effect to be studied. Unfortunately, there are many other ways for an atom to survive, most notably if it misses the laser beam completely. To overcome this problem, we must find some independent confirmation that the atom was ever in the presence of the intense light.

\section{WAVEPACKETS AND IONIZATION}

Intense field stabilization is closely tied to a different light-induced phenomenon in atoms -- wavepackets. Wavepackets are coherent superpositions of nondegenerate atomic states. Although nonstationary, they can have interesting and useful timevarying properties. The easiest type to make and visualize is the Kepler wavepacket. This is formed automatically when an atom is excited to to a series of Rydberg states with a pulse that is shorter than the classical Kepler period for the given $n$ state.

A Kepler wavepacket is similar to a classical orbiting electron, in the sense that the probability density makes periodic excursions from the region of the ion core to a distant radius of $\sim n^{2}$ atomic units, and back again in a time of $2 \pi n^{3}$ atomic unites. The dispersion in orbit times for adjacent $\mathrm{n}$ states eventually smears out this periodic behavior, but in some cases a periodic return to the core has been observed through many orbits. Since ionization requires the exchange of momentum with the ion, the ionization cross section is proportional to the amount of the wavefunction that overlaps the core. Orbiting wavepackets cause oscillations in this ionization rate.

\section{WAVEPACKETS AND STABILIZATION}

Wavepackets can be useful tools in atomic stabilization experiments. As an example, consider an atom which has just been excited into a Rydberg Kepler wavepacket by a short laser pulse.

5.1 Traversing Death Valley As the wavepacket travels to the outer part of its orbit, the ionization cross section becomes small. This is an ideal time to begin ramping up an intense laser pulse. The central $n$ value of the wavepacket can be adjusted so that the orbit time is comparable to the turn-on time of the intense laser. Thus, as the laser intensity passes through Death Valley, the electron is far from the nucleus so that it cannot exchange momentum necessary to ionize. In this way, the wavepacket can survive Death Valley. As the wavepacket approaches the ion for its next close encounter, the laser may already be intense enough to stabilize the state. 
5.2 Solving the Flop Out Problem When a Rydberg wavepacket becomes stabilized, other electrons in a multielectron atom may be less stable. The ionization of these other electrons will indicate that the atom in question experienced an intense field. Thus, although stabilization is primarily a one-electron phenomenon, the presence of additional electrons helps to solve the flop-out problem.

5.3 Dark wavepackets: a useful variation Wavepackets are not only created by exciting atoms with a short pulse from the ground or low-lying state into a superposition of Rydberg states. Another way to make a wavepacket is by ionizing (or rather, attempting to ionize) a single Rydberg state. We have already noted that there is a fundamental time scale associated with an atom -- its Kepler orbital period. No depletion of an atomic state can occur in times shorter than this. ${ }^{7}$

When a pulse incident on an atom has sufficient fluence to ionize the initial state, but insufficient duration, the ionization is necessarily incomplete. Only the part of the wave function near the nucleus can ionize. A new nonstationary state remains behind, which initially has similar probability density at large radii, but missing density near the nucleus. This state is a superposition of neighboring Rydberg states, just as a radial Rydberg wavepacket. In this case, however, the deficit is a "dark wavepacket."

Formation of a dark wavepacket is similar to formation of a Rydberg wavepacket from the ground state. In both cases, a laser pulse that is sufficiently short so that its bandwidth overlaps neighboring Rydberg states, is required. For the dark wavepacket, however, the neighboring states are not populated directly, but rather by the two-photon process of stimulated Raman scattering through the continuum. ${ }^{7}$

\section{BARIUM STARK STATES}

The formation of dark wavepackets that are superpositions of neighboring Rydberg states can be generalized to wavepacket formation within any spectrum of closely spaced energy eigenstates. One useful example is the formation of vibrational wavepackets in a molecule. Another example which is particularly important for stabilization experiments, is the formation of dark Stark wavepackets in an atom. Burnett et $\mathrm{al}^{8}{ }^{8}$ have analyzed the situation where Raman mixing processes between bound states and the continuum redistribute the atomic state into a superposition that cannot ionize. Their calculations can easily be extended to redistribution among $\mathrm{k}$ states in an initially populated Stark manifold.

We have performed wave-packet experiments in Barium atoms to test these ideas. ${ }^{7}$ We found that single photon ionization of the outer electron in a Ba 6snk Stark state could be nearly totally suppressed relative to high order ( $\geq 3$ photon) nonresonant multiphoton ionization of the tightly bound $6 \mathrm{~s}$ core electron. This population trapping occurs in intense short laser pulses, where the bandwidth of the ionizing laser is greater than the spacings between adjacent Stark states. 
Atomic barium is particularly interesting because its second electron could multiphoton ionize in our laser pulse, thus placing a tag on those atoms that experienced an intense laser field.

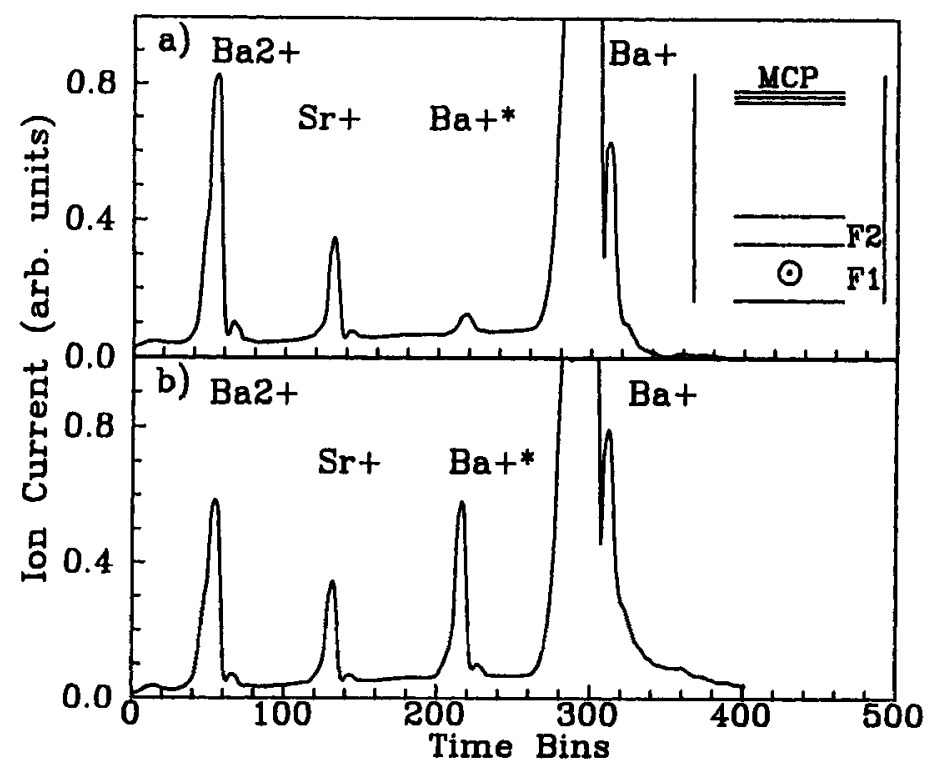

Fig 1. Time-of-Flight spectra showing the production of $\mathrm{Ba}^{2+}, \mathrm{Ba}^{+}$Rydberg states, and ground state $\mathrm{Ba}^{+}$ions using an $\mathrm{n}=25$ or $\mathrm{n}=35$ initial state for $1 \mathrm{a}$ and $1 \mathrm{~b}$, respectively. Both sets of data were taken with 162 $\mathrm{V} / \mathrm{cm}$ in the interaction region. The inset shows a schematic of the interaction region. (From reference 7)

The first step in the experiment was formation of a Barium Stark State. A thermal beam of ground state $\mathrm{Ba}$ atoms $\left(\rho \approx 10^{9} \mathrm{~cm}^{-3}\right.$, chamber base pressure $\approx 10^{-7}$ torr) in the presence of a static electric field of $F=\sim 100 \mathrm{~V} / \mathrm{cm}$, was excited in two steps from the $6 \mathrm{~s}^{2}$ ground state: first to the $6 \mathrm{~s} 6 \mathrm{p}$ resonance line using a $10 \mathrm{nsec}, 553.7 \mathrm{~nm}, 100$ $\mu \mathrm{J}$ dye laser; and then to a 6 snk Rydberg Stark state with $\mathrm{n}>20$ using a $10 \mathrm{nsec}, 420$ $\mathrm{nm}, 2 \mathrm{~mJ}$ dye laser. Here $\mathrm{k}$ is the parabolic quantum number associated with eigenstates of the atom in an electric field. Since the field mixes angular momentum states, all Stark states (not only the $\Delta l= \pm 1$ 6sns and 6snd states) can be reached in this way. There was sufficient laser fluence to deplete half of the ground state atoms in the interaction region.

Rydberg Stark states formed in this way were subjected to a focused (f/8) $70 \mathrm{psec}$, $10 \mathrm{~mJ}, 355 \mathrm{~nm}$ third harmonic of a mode-locked and amplified Nd:YAG laser. 
Afterwards the state of the atoms in the beam was examined to see whether Rydberg ions were present. These would indicate that the core electron, and not the Rydberg electron, had been ejected.

All ions were accelerated to a second field region with a much higher field, where any $\mathrm{Ba}^{+}$excited states were field ionized to form $\mathrm{Ba}^{2+}$. In this way, single ions, double ions, and Rydberg ions all had different drift velocities and could be distinguished. Two representative time-of-flight spectra are shown in Fig. 1. The prominent feature on the right is singly ionized $\mathrm{Ba}$ in its ground (or low-lying) state, which takes the longest time to exit the spectrometer. On the left is a smaller population of doubly ionized $\mathrm{Ba}$. Between these two $\mathrm{Ba}$ peaks are two more peaks representing singly ionized $\mathrm{Ba}$ Rydberg states, which have been field ionized during their flight to the detector, and an impurity ion, $\mathrm{Sr}^{+}$which commonly contaminates $\mathrm{Ba}$ metal. The $\mathrm{Ba}^{+*}$ signal indicates suppression of photoionization of the Stark Rydberg states.

Note that the increase in the population of the $\mathrm{Ba}^{+}$Rydberg states in figure $1 \mathrm{~b}$ is matched by a decrease in the $\mathrm{Ba}^{2+}$ signal. The suppression of ionization for the outer, Rydberg electron is clearly much greater in figure $1 \mathrm{~b}$. Let us examine why.

Figure la shows ionization of a Stark state with principal quantum number $\mathrm{n}=$ 25; Fig. $1 \mathrm{~b}$ shows a state with $\mathrm{n}=35$. The reason for the dramatic difference is the relative length of the laser pulse relative to the characteristic time for angular momentum mixing in the Stark states.

A 6snk Stark state $\mathrm{n}$ contains all angular momentum components with $\mathrm{l}<\mathrm{n}$; but the low angular momentum components contribute the most to photoionization. The fluence in our short laser pulse is $\approx 10^{3} \mathrm{~J} / \mathrm{cm}^{2}$, which is sufficient to one-photon ionize any state with cross section greater than $-500 \mathrm{~b}$. At this fluence we observe total ionization of $6 \mathrm{sns}$ and $6 \mathrm{snd}$ states for the $\mathrm{n}$ values studied, but $6 \mathrm{snf}$ and higher have cross sections that are two small.

The electric field couples 1 states, and so provides a mechanism for ionizing a Stark state through it's low 1 components; but this mixing has a time scale $\tau_{\mathrm{s}}$ which can be much longer than the laser pulse. Ionization of 6snk states is therefore suppressed if the ionizing laser pulse is shorter than this characteristic mixing time between 1 states.

The characteristic rate $2 \pi / \tau_{\mathrm{s}}$ for mixing within a Stark state just the energy splitting between neighboring Stark states, divided by $\bar{h}$. This rate is $3 F n$, where $F$ is the applied electric field in atomic units. ${ }^{8}$ A laser pulse much shorter than $\tau_{\mathrm{s}}$ can only couple low angular momentum components of the Stark state to the continuum. It therefore leaves behind a "trapped" nonstationary state in the atom consisting of high 1 orbitals. This evolves in time like a parabolic wavepacket, ${ }^{9}$ in precise analogy to the Kepler orbital wavepacket described above.

The results shown in Fig. 1 are now easily understood. The field in the interaction region is $\mathrm{F} 1=162 \mathrm{~V} / \mathrm{cm}$. The $\mathrm{n}=25$ state shown in Fig. 1a has a Stark mixing time, $\tau_{\mathrm{s}}=60 \mathrm{psec}$ which is slightly less than the laser pulse duration; and the $\mathrm{n}=35$ state of 
Fig. $1 \mathrm{~b}$ has a mixing time, $\tau_{s}=230 \mathrm{psec}$ which is much longer than the laser pulse. The probability of finding the electron in a low angular momentum state is nearly unity in Fig. 1a and is almost negligible in Fig. $1 \mathrm{~b}$.

This trapping has some features in common with stabilization; however, it is important to note that the suppression of ionization is induced by the short timescale of the laser pulse, as much as by the saturation of the ionization out of low 1 states. Nonetheless, this experimental technique can be used to search for high intensity stabilization. The formation of a Rydberg wavepacket is a convenient way to "shelve" the outer electron during a time when the laser pulse is rising in intensity.

\section{WAVEPACKETS DURING ATI EXPERIMENTS IN RARE GASES}

ATI in atoms is greatly enhanced when one or more excited bound states have energies which lie an integral number of photons above the ground state. ${ }^{9}$ This is not merely a matter of coincidence. Because intensity-dependent AC Stark shifts can be on the order of the photon energy, multiphoton resonances will occur for almost every state in the atom at some time during the laser pulse. As each Rydberg state passes through resonance, there is some probability that population will be trapped in it. This, then, is another form of trapping that depends not on the duration of the laser pulse, but rather on the duration of the transient multiphoton resonance, which can be much shorter.

This type of high intensity trapping can be present in any ATI experiment. Rare gas atoms have been most commonly used as laser targets, because the relatively large ionization potentials (12-25 eV) and lack of low lying excited states enable them to survive to high intensities before ionizing. Under these conditions, the AC Stark shifts between the ground and excited states are comparable to the Ponderomotive Potential. For example, a $\mathrm{Kr}$ atom (I.P. $=14.0 \mathrm{eV}$ ) exposed to $\lambda=0.77 \mu \mathrm{m}$ radiation $(\mathrm{h} v=1.6 \mathrm{eV})$ requires nine photons to ionize at low intensity. When the intensity exceeds $1.9 \times 10^{13} \mathrm{~W} / \mathrm{cm}^{2}$, however, high-lying Rydberg states can be resonantly excited with nine photons, and ionization proceeds through the absorption of one additional photon.

Until recently, evidence for ionization enhancement due to AC Stark shifted resonances came entirely from ATI photoelectron spectra. ${ }^{3}$ Of course, resonant enhancement does not equate to trapping of population. It may be that in most cases, single photon ionization of the Rydberg states occurs immediately in the intense laser light required to drive the nine-photon absorption.

There are several circumstances, however, where population is transferred from the ground state to Rydberg states in a strong laser field. Several recent experiments have investigated this.

The most straightforward test is an ordinary ATI experiment, where the state of the atoms is examined afterward to see if there are any excited states left over. In this way, we have recently observed population trapping in highly excited Rydberg states $(\mathrm{n}>14)$ in $\mathrm{Kr}$ and $\mathrm{Xe}$ atoms exposed to laser intensities above $5 \times 10^{15} \mathrm{~W} / \mathrm{cm}^{2}$. 


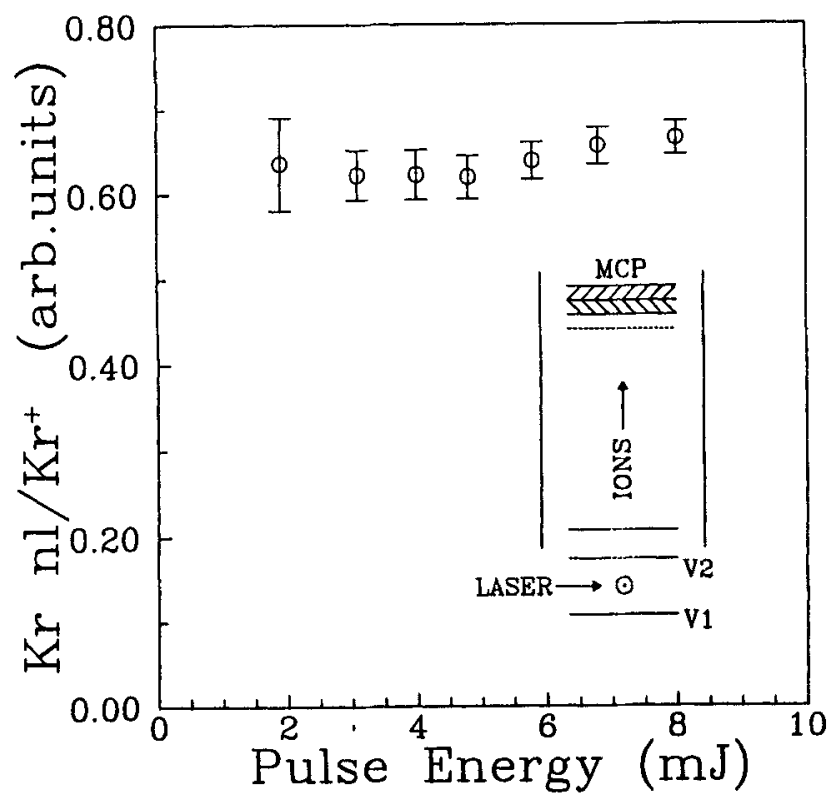

Figure 2. Ratio of $\mathrm{Kr}$ Rydberg states observed to $\mathrm{Kr}^{+}$ions as a function of laser pulse energy for a fixed temporal pulse width. The actual ratio has a constant value $<1 \%$. Inset: Schematic of the laser/atom interaction region. (From reference 10.)

7.1 Krypton experiment ${ }^{10}$ We used $770 \mathrm{~nm} 100 \mathrm{fsec}$ laser pulses, focused into a chamber with a low density $\left(\sim 1 \times 10^{10} \mathrm{~cm}^{-3}\right)$ of krypton. The laser was focused between two parallel field plates as shown in the inset in Fig. 2. The total ion yield could be collected with a small electric field applied after the laser pulse. Alternatively, if the field was applied backwards, ions could be accelerated into the lower conducting plate. Once these ions were removed, a large pulsed electric field in the forward direction could ionize remaining neutral Rydberg states with $n>14$, and push them toward a detector. In this way, only ions formed by the field ionization of excited neutral atoms were detected.

Fig. 2 shows the ratio of Rydberg states to $\mathrm{Kr}^{+}$vs. laser pulse energy, for constant pulsewidth $\left(\tau_{L} \sim 100 \mathrm{fsec}\right)$. The ratio has a constant value of less than $1 \%$. At these intensities, both the $\mathrm{Kr}^{+}$signal and the Rydberg excitation cross section are saturated, so they are both proportional to the focal volume as the pulse energy increases.

Fig. 3 shows the same ratio, but as a function of the laser pulse duration at fixed pulse fluence. The pulse width was increased by adjusting the grating spacing in the pulse compressor away from maximum compression. The ratio of the Rydberg signal 


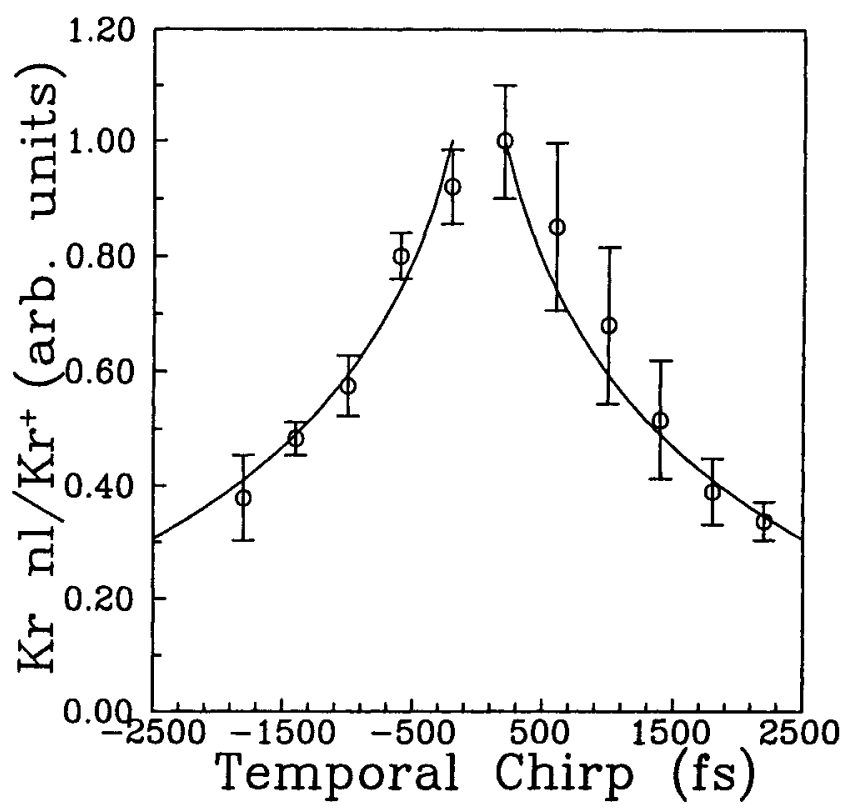

Figure 3. Same ratio as in Fig. 2 plotted as a function of temporal chirp in the laser pulse. The chirp was created by introducing positive (+ sign) or negative (- sign) group velocity dispersion in the laser pulse using a diffraction grating. The decrease in the ratio for a broad temporal pulse is due to the length of the laser pulse and not to a decrease in the laser intensity. The curve is described in the text. (From reference 10.)

to the $\mathrm{Kr}^{+}$ion signal is almost identical for grating spacings that are longer than optimum, or shorter, corresponding to positive and negative dispersion. Evidently the sense of the frequency chirp has no effect on the Rydberg population in this case, but the length of the pulse is very important: far more Rydberg states are present when the pulse is short. Such a pulse-width dependence suggests wavepacket formation.

The solid curve is the predicted signal if the ionization fraction is strictly proportional to the amount of time that the Rydberg electron spends near the ion core during its classical orbit. We expect this result if the laser fluence is high enough to saturate the ionization cross section, but its duration is less than the classical Kepler orbit time. Then the probability for photoionization of an $n$ state is the ratio of the laser pulse duration, $\tau_{\mathrm{L}}$ to the Kepler period $\tau_{\mathrm{k}}=2 \pi \mathrm{n}^{3}$. $^{11,12}$

The result should be quite different if the intensity is too low to saturate the ionization process. This may be the case in our studies of Xe, where we find the trapped fraction decreases with increasing laser intensity. When these measurements at 


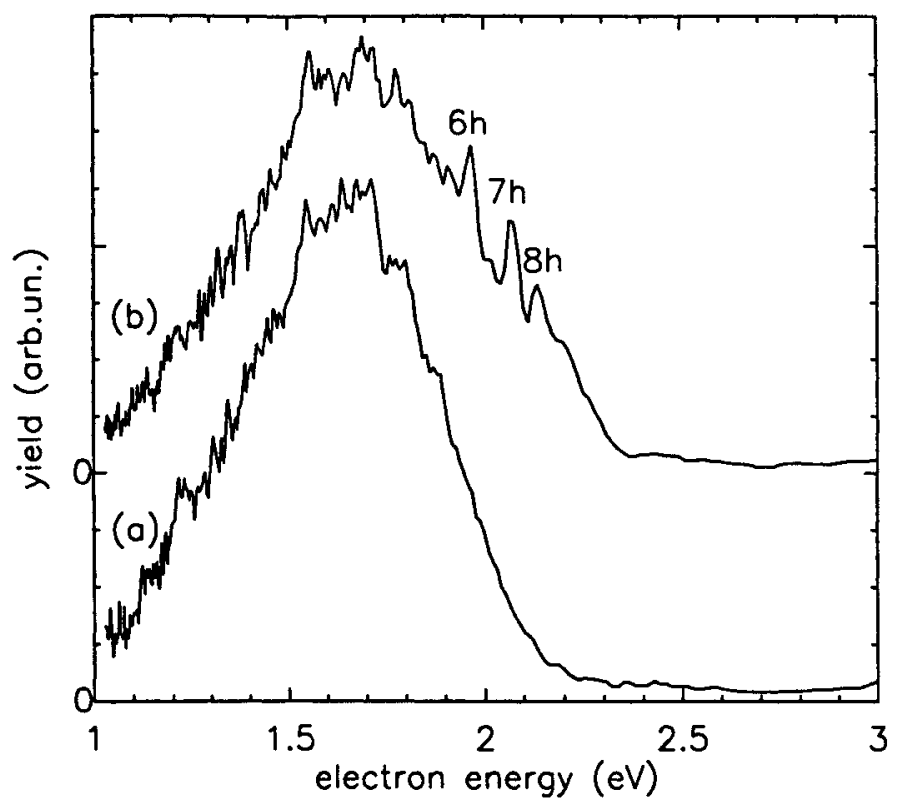

Figure 4. Photoelectrons from xenon ionized by circularly polarized 100 fsec light pulses, from the experiment of de Boer, reference 13. In the top figure, a second laser is fired after the pulse to ionize any remaining excited state population. $6 \mathrm{~h}, 7 \mathrm{~h}$, and $8 \mathrm{~h}$ excited states are clearly visible as additional sharp peaks in the top spectrum, proving that the short pulse was able to excite these states. (From reference 13).

constant pulse duration are used to remove the intensity dependence from the measurements at constant pulse energy, the production of Xe Rydberg states again shows a pulse duration dependence similar to $\mathrm{Kr}$.

\section{TWO-LASER EXPERIMENTS}

8.1 Linear Polarization These Xe results may help to interpret another series of experiments carried out by de Boer and coworkers. ${ }^{13}$ They have observed that up to $50 \%$ of Rydberg states ( $<8$ ) created during ATI of Xe by a 100 fsec laser pulse remain trapped. Their experiments are performed in a regime where it is possible to excite substantial population to these trapped states, without saturating the single photon cross section to the continuum.

These experiments utilized a second, nanosecond pulse laser as a probe of the excited state population produced by the sub-picosecond ionizing pulse. The second laser had a different color, so when it ionized an excited state it produced 

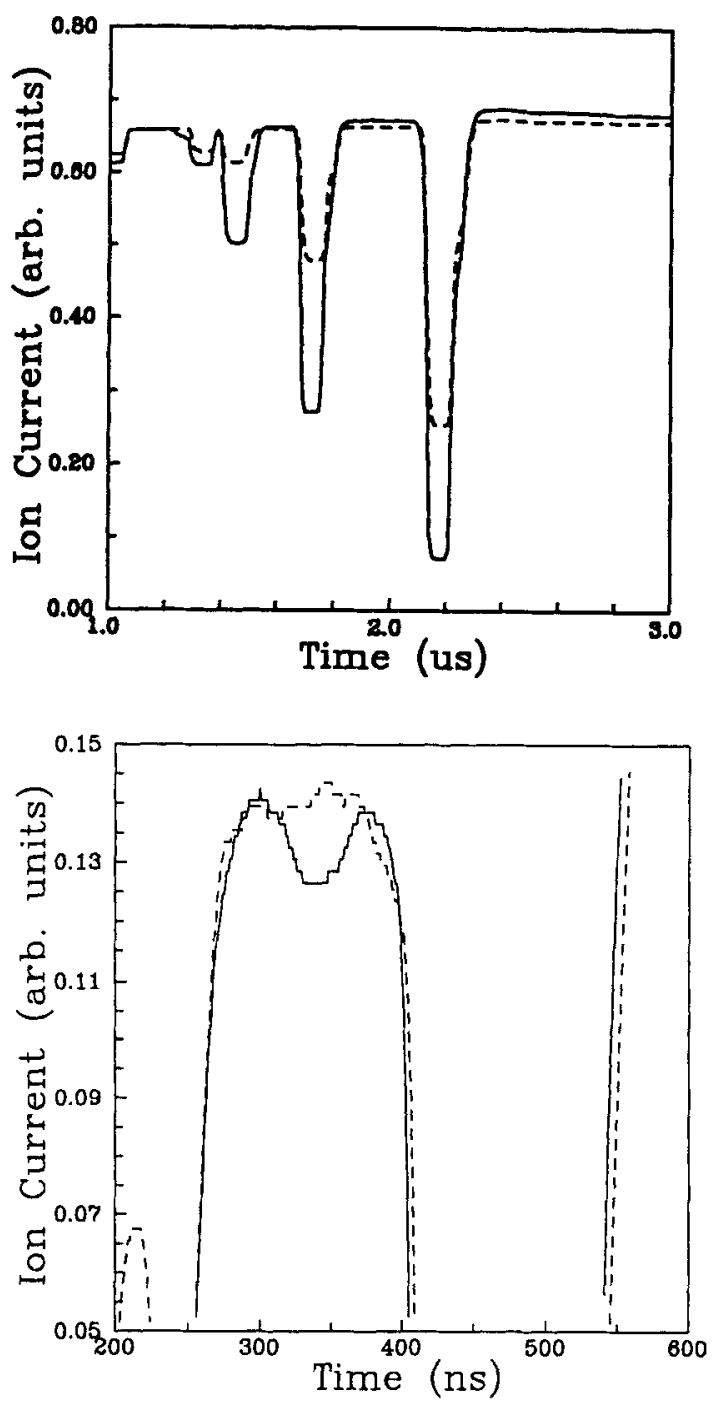

Figure 5. Top: Time-of-flight spectrum for multiple charge states of xenon ionized by $100 \mathrm{fsec} 770 \mathrm{~nm}$ light pulses. The time-of-flight apparatus is similar to the one shown in figure 2 . The solid line is for linear polarization, and the dashed line is for circular polarization. After the pulse, some ions are left in excited states. These appear as counts in the detector between the arrival times of the ground state ions. Bottom: This is an expanded view of the region between $\mathrm{Xe}^{2+}$ and $\mathrm{Xe}^{3+}$, showing Rydberg states of $\mathrm{Xe}^{2+}$. If circular polarization is used (dashed line), no excited states are formed. 
photoelectrons with energies that were separated from the ATI spectral peaks.

8.2 Circular Polarization The most dramatic trapping effects seen in the FOM experiments occurred for circular polarization. It has long been observed that circular polarization does not produce resonant enhancements in ATI peaks. ${ }^{14}$ Even before the short pulse resonant enhancement peaks were discovered, it was noted that ATI with circular polarization was strongly suppressed by the poor overlap of the high angular momentum states in the continuum and the ground state. ${ }^{15}$ The FOM group were able to demonstrate that bound high angular momentum states can be populated through nonlinear high order multiphoton absorption, even though the absorption of one additional circular photon to reach the continuum is nearly impossible. Figure 4 shows the $6 \mathrm{~h}, 7 \mathrm{~h}$, and $8 \mathrm{~h}$ states in xenon trapped in this way. Unfortunately, this behavior is not expected to occur in high l- limit (see next section).

\section{TRAPPED STATES IN MULTIPLY IONIZED ATOMS}

Finally, trapped Rydberg states can be observed in multiply ionized species as well. Figure 5 shows data from our experiments in xenon, irradiated by linearly polarized $770 \mathrm{~nm}$ light, where it takes 20 photons to ionize the ground state. Small amounts of $\mathrm{Xe}^{2+} *$ are clearly present in the ion spectra. If the light is circularly polarized, no bound state are formed, since the multiphoton excitation of very high angular momentum states $(1>18)$ is highly suppressed. We have detected Rydberg states in multiply ionized $\mathrm{Kr}$ and $\mathrm{Xe}$, with energies up to $32 \mathrm{eV}$ above the ionic ground state, requiring at least 20 photon absorption.

\section{CONCLUSIONS}

Atoms can be bound by the mutual interaction of an intense laser and a static Coulomb field. Such systems represent a new class of quantum states, with some interesting properties. Several mechanisms have now been proposed for the trapping and stabilization of bound states in atoms during intense field ionization. So far, there is no experimental evidence for adiabatic stabilization, such as "dichotomous" states; ${ }^{11}$ but much progress has been made in overcoming the technical obstacles to such an observation. In addition, various dynamical trapping mechanisms, such as the formation of non-ionizing wavepackets, have been discovered and studied.

\section{REFERENCES}

1. P. Agostini, F. Fabre, G. Mainfray, G. Petite, and N. Rahman, Phys. Rev. Lett. 42, 1127 (1979); P. Kruit, J. Kimman, H. G. Muller, and J. J. van der Wiel, Phys. Rev. A28, 248 (1983).

2. M. Pont and M. Gavrilla, Phys. Rev. Lett. 65, 2362 (1990). M. Gavrilla and J.Z. Kominski, Phys. Rev. Lett. 52, 613 (1984). 
498 Stability of Atoms in Intense Laser Fields

4. For a review of this, see P.H. Bucksbaum, Atomic Physics 11 , ed. by S. Haroche, J.C. Gay, and G. Grynberg, pp.311-325 (World Scientific, Singapore, 1989).

5. John A. Yeazell and C.R. Stroud, Jr., Phys. Rev. Lett., 60, 1494 (1988).

6. L.D. Noordam, A. ten Wolde, A. Lagendijk, and H.B. van Linden van den Heuvell, Phys. Rev. A 40, 6999 (1989).

7. 5. R.R. Jones and P.H. Bucksbaum, Phys. Rev. Lett. 67, 3215 (1991).

8. K. Burnett, P.L. Knight, B.R.M. Piraux, and V.C. Reed, Phys. Rev. Lett. 66,301 (1991).

9. R.R. Freeman, P.H. Bucksbaum, H. Milchberg, S. Darack, D. Schumacher, and M.E. Geusic, Phys. Rev. Letters 59, 1092 (1987).

10. R.R. Jones, D.W. Schumacher, and P.H. Bucksbaum, preprint, (1992).

11. H.A. Bethe and E. Salpeter, Quantum Mechanics of One and Two Electron Atoms, (Plenum, New York, 1977).

12. H. Stapelfeldt, D.G. Papaiounnou, L.D. Noordam, and T.F. Gallagher, Phys. Rev. Lett. 67, 3223 (1991).

13. M.P. de Boer and H.G. Muller, Phys. Rev. Lett., 68, 2747 (1992); M.P. de Boer, L.D. Noordam and H.G. Muller, preprint (1992).

14. P.H. Bucksbaum, L.D. Van Woerkom, R.R. Freeman, and D.W. Schumacher, Phys. Rev. A 41, 4119 (1990).

15 . P. H. Bucksbaum, M. Bashkansky, R. R. Freeman, T. J. Mcllrath, and L. F. DiMauro, Phys. Rev. Lett. 56, 2590 (1986). 